Sādhanā Vol. 38, Part 4, August 2013, pp. 557-569. (C) Indian Academy of Sciences

\title{
Flexibility-based structural damage identification using Gauss-Newton method
}

\author{
B CHEN $^{\mathrm{a}}$ and S NAGARAJAIAH ${ }^{\mathrm{a}, \mathrm{b}, *}$ \\ ${ }^{a}$ Department of Civil and Environmental Engineering, Rice University, Houston, \\ TX 77005, USA \\ ${ }^{\mathrm{b}}$ Department of Mechanical Engineering and Materials Science, Rice University, \\ Houston, TX 77005, USA \\ e-mail: chenbl@rice.edu; nagaraja@rice.edu
}

MS received 9 October 2011; revised 17 May 2013; accepted 26 May 2013

\begin{abstract}
Structural damage will change the dynamic characteristics, including natural frequencies, modal shapes, damping ratios and modal flexibility matrix of the structure. Modal flexibility matrix is a function of natural frequencies and mode shapes and can be used for structural damage detection and health monitoring. In this paper, experimental modal flexibility matrix is obtained from the first few lower measured natural frequencies and incomplete modal shapes. The optimization problem is then constructed by minimizing Frobenius norm of the change of flexibility matrix. Gauss-Newton method is used to solve the optimization problem, where the sensitivity of flexibility matrix with respect to structural parameters is calculated iteratively by only using the first few lower modes. The optimal solution corresponds to structural parameters which can be used to identify damage sites and extent. Numerical results show that flexibility-based method can be successfully applied to identify the damage elements and is robust to measurement noise.
\end{abstract}

Keywords. Structural damage detection; flexibility; Gauss-Newton method.

\section{Introduction}

Existence of structural damages in civil infrastructure, such as high-rise buildings, long-span bridges, offshore platforms, etc., may greatly influence the overall performance of the system or even lead to disastrous consequences. Therefore, detecting structural damage caused by earthquakes, hurricanes, or explosions immediately after the event or monitoring long-term deterioration is necessary for remedy or repairs.

In the last few decades, many methods have been proposed for structural damage detection, for example, the literature review by Doebling et al (1996) and Sohn et al (2003). Visual inspection is widely used in bridges and buildings today because it is easy and straightforward in

*For correspondence 
some sense. Other 'local' methods, such as acoustic emission, ultrasonic and eddy current scanning, X-ray inspection, etc., provide other options for structural damage detection. However, in more complex structures, structural damage may be hidden for visual inspection or local testing. Therefore, the conventional visual inspection or 'local' methods may not work or will be timeconsuming and costly. Instead, structural dynamic characteristics, such as modal shapes, natural frequencies, stiffness matrix and flexibility matrix, provide a 'global' approach to evaluate the structural state because the presence of structural damage will change such dynamic characteristics of the structure. This leads to the field of modal-based structural damage detection and health monitoring.

Modal-based structural damage detection methods have been extensively studied in the last decades (Liu 1995; Shi et al 2000a; Ricci 2000; Ren \& Roeck 2002). Liu (1995) used the measured natural frequencies and modal shapes to formulate the eigenequation of the truss, whose norm is minimized by an optimization program for structural identification and damage detection. Shi et al (2000a) applied the sensitivity and statistical-based method called the multiple damage location assurance criterion (MDLAC) to the incomplete measured modal shapes to localize the damage sites and then used measured natural frequencies to determine the damage extent. Ricci (2000) located structural damages by computing the Euclidean distances between the measured mode shapes and the best achievable modal eigenvectors. Ren \& Roeck (2002) demonstrated that multiplying the damaged eigenvalue equations with the damaged modes or undamaged modes provides more equations than the strain energy-based method, which will guarantee the damage localization. These equations are solved by non-negative least-squares technique. All of the above methods use either measured natural frequencies, or measured complete (or incomplete) modal shapes, or both to identify structural damages. However, they did not combine natural frequencies and modal shapes together to form only one quantity so that the sensitivity of natural frequencies and mode shapes with respect to structural damage index can be used simultaneously for structural damage detection.

Recently, the modal flexibility matrix has gained much attention in the field of structural damage detection and finite element model updating, since, it is the function of structural natural frequencies and mode shapes and will more readily indicate changes in stiffness than either natural frequencies or mode shapes (Zhao \& DeWolf 1999). Meanwhile, the modal flexibility matrix is easy to obtain experimentally, since, only a few lower modes are required to achieve reasonable accuracy. Pandey \& Biswas (1994) determined damage sites directly using changes in the flexibility matrix of a structure. Bernal $(2002,2006)$ presented the concept of damage locating vectors (DLVs), which are associated with sensor coordinates and can be computed systematically as the null space of the change in measured flexibility. When statically applied to the structure, DLVs will induce stress fields whose magnitude is zero in the damaged elements. Those elements are thought of as potentially damaged elements. Wu \& Law (2004) studied the truncated modal flexibility sensitivity with respect to the generic parameters and proposed a model updating method based on this sensitivity and incomplete measurements. Jaishi \& Ren (2006) presented a sensitivity-based finite element model updating for damage detection. The objective function consisting of the modal flexibility residual is formulated and then trust region method is used to find the optimal structural parameters for damage detection and model updating. However, if the number of Degree of Freedom (DOF) is too large to install a full set of sensors, how to effectively and efficiently apply the flexibility-based method is still a challenging problem.

In this paper, the flexibility-based method and Gauss-Newton optimization algorithm are combined to detect structural damage. Firstly, the experimental flexibility matrix corresponding to the measured DOFs is obtained by using the first few lower incomplete modes. Secondly, the 
Frobenius norm of the change of flexibility matrix is minimized by Gauss-Newton algorithm. The solution of the minimization problem corresponds to structural damage index and can be used to quantify structural damage extent. The sensitivity matrix of flexibility with respect to structural parameters is formed using the first few lower modes at the measured DOFs. GaussNewton method is a very effective and efficient method for nonlinear unconstrained optimization problem. It does not need Hessian matrix computation, which is complex and computationally expensive. Simulation examples demonstrate that the proposed flexibility-based method can detect and identify structural damages successfully.

\section{Theoretical background}

\subsection{Gauss-Newton method}

Consider the following unconstrained minimization problem

$$
\min f(\mathbf{x})=\frac{1}{2} \sum_{i=1}^{m}\left[y(\mathbf{x})-y_{i}\right]^{2}
$$

where the variable $\mathbf{x}=\left(x_{1}, x_{2}, \ldots, x_{n}\right)^{T} \in \mathbf{R}^{n}, n<m ; y(\mathbf{x})$ is the nonlinear function of $\mathbf{x} ; y_{i}$ is the measured value. The coefficient $1 / 2$ is only for the convenience of computation. It does not influence the solution of the minimization problem. Define the function $\varphi_{i}(\mathbf{x})$ and $F(\mathbf{x})$ as

$$
\begin{gathered}
\varphi_{i}(\mathbf{x})=y(\mathbf{x})-y_{i}, \quad i=1,2, \cdots m \\
F(\mathbf{x})=\left[\varphi_{1}(\mathbf{x}), \varphi_{2}(\mathbf{x}), \cdots, \varphi_{m}(\mathbf{x})\right]^{T} .
\end{gathered}
$$

Substituting Eqs (2) and (3) into Eq. (1) yields

$$
\min f(\mathbf{x})=\frac{1}{2} \sum_{i=1}^{m}\left[\varphi_{i}(\mathbf{x})\right]^{2}=\frac{1}{2} F(\mathbf{x})^{T} F(\mathbf{x}) .
$$

So, the optimal solution $\mathbf{x}$ is achieved when $\nabla f(\mathbf{x})=F^{\prime}(\mathbf{x})^{T} F(\mathbf{x})=0$.

Starting from an initial point $\mathbf{x}_{0}$, we need to construct an updating algorithm for $\mathbf{x}$ such that the objective function $f(\mathbf{x})$ approaches the minimal value quickly. Newton's method may be the most straightforward method and it converges very fast to the correct value. It updates the variable $\mathbf{x}$ in the following form

$$
\mathbf{x}_{k+1}=\mathbf{x}_{k}-H\left(\mathbf{x}_{k}\right)^{-1} \nabla f\left(\mathbf{x}_{k}\right)=\mathbf{x}_{k}-H\left(\mathbf{x}_{k}\right)^{-1} F^{\prime}(\mathbf{x})^{T} F(\mathbf{x}),
$$

where $H\left(\mathbf{x}_{k}\right)$ is the Hessian matrix of the objective function $f(\mathbf{x})$, which is evaluated at $\mathbf{x}_{k}$. The Jacobi matrix of $F(\mathbf{x})$ is defined as

$$
F^{\prime}(\mathbf{x})=\left[\begin{array}{cccc}
\frac{\partial \varphi_{1}(\mathbf{x})}{\partial x_{1}} & \frac{\partial \varphi_{1}(\mathbf{x})}{\partial x_{2}} & \cdots & \frac{\partial \varphi_{1}(\mathbf{x})}{\partial x_{n}} \\
\frac{\partial \varphi_{2}(\mathbf{x})}{\partial x_{1}} & \frac{\partial \varphi_{2}(\mathbf{x})}{\partial x_{2}} & \cdots & \frac{\partial \varphi_{2}(\mathbf{x})}{\partial x_{n}} \\
\vdots & \vdots & \ddots & \vdots \\
\frac{\partial \varphi_{m}(\mathbf{x})}{\partial x_{1}} & \frac{\partial \varphi_{m}(\mathbf{x})}{\partial x_{2}} & \cdots & \frac{\partial \varphi_{m}(\mathbf{x})}{\partial x_{n}}
\end{array}\right] \in R^{m \times n}
$$


Though Newton method, as expressed in Eq. (5), has many advantages, it suffers from two serious disadvantages from the point of view of practical calculation: One is the difficulty of computing Hessian matrix $H\left(\mathbf{x}_{k}\right)$ at each iteration step; and the other disadvantage is the fact that without some modifications, its inverse may not exist. In order to overcome the above disadvantages, many modification methods have been proposed. Gauss-Newton method is one of modified Newton's methods and will be briefly introduced as follows. Expanding $F(\mathbf{x})$ at $\mathbf{x}_{k}$ and ignoring the higher terms lead to

$$
F(\mathbf{x}) \approx F\left(\mathbf{x}_{k}\right)+F^{\prime}\left(\mathbf{x}_{k}\right)\left(\mathbf{x}-\mathbf{x}_{k}\right) .
$$

Substituting Eq. (7) into $\nabla f(\mathbf{x})=F^{\prime}(\mathbf{x})^{T} F(\mathbf{x})$, we obtain

$$
F^{\prime}\left(\mathbf{x}_{k}\right)^{T}\left[F\left(\mathbf{x}_{k}\right)+F^{\prime}\left(\mathbf{x}_{k}\right)\left(\mathbf{x}-\mathbf{x}_{k}\right)\right]=0 .
$$

$\mathbf{x}$ can be solved from the above equation and set as next iteration value,

$$
\mathbf{x}_{k+1}=\mathbf{x}_{k}-G\left(\mathbf{x}_{k}\right)^{-1} \nabla f\left(\mathbf{x}_{k}\right),
$$

where

$$
G(\mathbf{x})=F^{\prime}(\mathbf{x})^{T} F^{\prime}(\mathbf{x}) .
$$

In order to avoid singular or ill-conditioned $G(\mathbf{x})$, a damping term is usually added to $G(\mathbf{x})$

$$
\widehat{G}(\mathbf{x})=G(\mathbf{x})+\mu I,
$$

where the damping coefficient $\mu>0$, which guarantees that $\widehat{G}(\mathbf{x})$ is symmetric and positive definite. Therefore, the iteration process becomes

$$
\mathbf{x}_{k+1}=\mathbf{x}_{k}-\widehat{G}\left(\mathbf{x}_{k}\right)^{-1} \nabla f\left(\mathbf{x}_{k}\right) .
$$

The stopping criterion is usually selected as

$$
\frac{\left|f\left(\mathbf{x}_{k+1}\right)-f\left(\mathbf{x}_{k}\right)\right|}{1+\left|f\left(\mathbf{x}_{k}\right)\right|}<\varepsilon,
$$

where $\varepsilon$ is a very small positive number.

\subsection{Modal flexibility-based structural damage detection}

There is an inverse relationship between modal flexibility and the square of natural circular frequencies. Thus, high frequency modes contribute little to modal flexibility and can be truncated without introducing significant error. This property indicates a great potential for modal flexibility-based structural damage detection because it is quite challenging to experimentally extract high modes of a structure. Modal flexibility can be calculated from a small number of lower modes with a reasonable accuracy. For this reason, structural damage detection based on modal flexibility has gained increasing attention recently.

Consider an undamped free vibration of motion of a linear structure

$$
M \ddot{X}(t)+K X(t)=0,
$$


where $X(t) \in \mathbf{R}^{n}$ is the displacement. $M$ and $K$ are the mass and stiffness matrices, respectively. Assume that $\omega_{i}$ and $\phi_{i}$ are the $i$ th natural circular frequency and mass-normalized mode shape of the system, respectively. They satisfy the following relations

$$
\begin{gathered}
K \phi_{i}=\omega_{i}^{2} M \phi_{i} \\
\phi_{i}^{T} M \phi_{i}=1 .
\end{gathered}
$$

Set $\Phi=\left[\phi_{1}, \phi_{1}, \ldots, \phi_{n}\right]^{T}$ and $\Lambda=\operatorname{diag}\left(\omega_{1}^{2}, \omega_{2}^{2}, \ldots, \omega_{n}^{2}\right)$, then we have

$$
\begin{aligned}
& K \Phi=M \Phi \Lambda \\
& \Phi^{T} M \Phi=I .
\end{aligned}
$$

Pre-multiplying Eq. (17) by $\Phi^{T}$ and using the fact of Eq. (18) lead to

$$
\Phi^{T} K \Phi=\Lambda \text {. }
$$

Then the stiffness matrix $K$ can be expressed in terms of

$$
K=\Phi^{-T} \Lambda \Phi^{-1}
$$

Therefore, flexibility matrix of the system is

$$
F=K^{-1}=\Phi \Lambda^{-1} \Phi^{T}=\sum_{i=1}^{n} \phi_{i} \frac{1}{\omega_{i}^{2}} \phi_{i}^{T} .
$$

Equation (21) indicates that the influence of the modes on the flexibility matrix $F$ decreases with $\omega^{2}$. Only a few lower modes are required to achieve reasonable accuracy in the flexibility matrix $F$. This fact is practically significant, since, the first few lower modes are easy to obtain during the vibration test. Suppose that the first $p(<n)$ modes are measured; and the rest $q(=n-p)$ higher modes are unknown and will be ignored. Thus, the flexibility matrix $F$ can be partitioned as

$$
F=F_{p}+F_{q} \approx F_{p}
$$

where $F_{p}$ is the modal flexibility matrix, computed from measured modes as

$$
F_{p}=\sum_{i=1}^{p} \phi_{i} \frac{1}{\omega_{i}^{2}} \phi_{i}^{T}
$$

and $F_{q}$ is the residual flexibility matrix, computed from the residual modes as

$$
F_{q}=\sum_{i=p+1}^{n} \phi_{i} \frac{1}{\omega_{i}^{2}} \phi_{i}^{T} .
$$

In practice, the full degree-of-freedom system is so large that it is impractical to install as many sensors as structural degree of freedom. Only a limited number of measurements are available. If 
we partition the full DOF set into measured and non-measured DOFs and multiply the partitions, it is shown by Doebling (1995) that

$F_{p}=\sum_{i=1}^{p} \phi_{i} \frac{1}{\omega_{i}^{2}} \phi_{i}^{T}=\sum_{i=1}^{p}\left(\frac{\phi_{i m}}{\phi_{i u}}\right) \frac{1}{\omega_{i}^{2}}\left(\phi_{i m}^{T}: \phi_{i u}^{T}\right)=\left(\begin{array}{c:c}\sum_{i=1}^{p} \phi_{i m} \frac{1}{\omega_{i}^{2}} \phi_{i m}^{T} & \sum_{i=1}^{p} \phi_{i m} \frac{1}{\omega_{i}^{2}} \phi_{i u}^{T} \\ \hdashline \sum_{i=1}^{p} \phi_{i u} \frac{1}{\omega_{i}^{2}} \phi_{i m}^{T} & \sum_{i=1}^{p} \phi_{i u} \frac{1}{\omega_{i}^{2}} \phi_{i u}^{T}\end{array}\right)=\left(\begin{array}{c:c}F_{p}^{m m} & F_{p}^{m u} \\ \hdashline F_{p}^{u m} & F_{p}^{u u}\end{array}\right)$

where $F_{p}^{m m}$ is called the measured flexibility matrix; $\phi_{i m}$ and $\phi_{i u}$ are, respectively, the measured modal shapes of the structure at the measured and unmeasured DOFs. The measured flexibility matrix $F_{p}^{m m}$ can be obtained directly from experiment results and is used to detect structural damages thereafter. For the sake of simplicity and clarity, the measured flexibility matrix $F_{p}^{m m}$ will be referred as $F_{\text {exp }}$ and the corresponding analytical flexibility matrix $F_{p}^{m m}$ as $F_{\text {ana. }}$.

Since the flexibility matrix is the function of natural frequencies and mode shapes of the structure, and the natural frequencies and mode shapes depends on structural properties, the flexibility matrix is thus related to structural parameters. Therefore, the change of structural parameters will change the flexibility matrix. The objective of this paper is to design a mathematical algorithm, which can identify structural parameters, such that the analytical flexibility matrix $F_{\text {ana }}$ is as close as possible to the measured flexibility matrix $F_{\text {exp }}$. The minimization problem can be defined as

$$
\min f(x)=\frac{\left\|F_{\text {ana }}-F_{\text {exp }}\right\|_{F}^{2}}{2\left\|F_{\exp }\right\|_{F}^{2}}
$$

where $\|\cdot\|$ represents the Frobenius norm of the matrix, for example the Frobenius norm of matrix $A \in \mathbf{R}^{m \times n}$ is

$$
\|A\|_{F}=\sqrt{\operatorname{trace}\left(\mathrm{A}^{\mathrm{T}} \mathrm{A}\right)}=\sqrt{\sum_{i=1}^{m} \sum_{j=1}^{n} A_{i j}^{2}} .
$$

Then Eq. (26) can be expressed as

$$
\min f(x)=\frac{1}{2\left\|F_{\text {exp }}\right\|_{F}^{2}}\left[\sum_{i=1}^{m} \sum_{j=1}^{m}\left(F_{\text {ana }}-F_{\text {exp }}\right)_{i j}^{2}\right] .
$$

The structural damages may be represented in many ways: loss of mass in some elements, connection failure in some joints, presence of crack and so on. Since the mass of the system will usually remain intact during the dynamic process, only changes in the stiffness matrix of the structure are assumed in this paper. Meanwhile, the stiffness changes will not change the connectivity of the structure and affect significantly the damping properties of the structure. The stiffness matrix of the structure is assumed as a summation of stiffness matrix of each element of the structure

$$
K=\sum_{j=1}^{N_{e}} \alpha_{j} K_{j}^{e}
$$

where $N_{e}$ is the number of elements; $K_{j}^{e}$ is the stiffness matrix for the $j$ th element; $\alpha_{j}$ is the damage indication factor for the $j$ th element, which ranges from 0 to 1 . When $\alpha_{j}$ is equal to 1 , it 
means the structure is healthy; whereas it is zero, it represents the stiffness of the corresponding element is totally damaged. Therefore, the optimization problem (Eq. 28) is actually a constraint optimization problem

$$
\begin{aligned}
& \min f(x)=\frac{1}{2\left\|F_{\mathrm{exp}}\right\|_{F}^{2}}\left[\sum_{i=1}^{m} \sum_{j=1}^{m}\left(F_{\mathrm{ana}}(\alpha)-F_{\mathrm{exp}}\right)_{i j}^{2}\right] \\
& \text { s.t. } 0 \leq \alpha_{j} \leq 1, \quad j=1,2, \cdots, N_{e},
\end{aligned}
$$

where $\alpha=\left[\alpha_{1}, \alpha_{2}, \ldots, \alpha_{N e}\right]^{T}$.

Gauss-Newton method cannot be used directly to solve the above constrained optimization problem. We need to convert it to the unconstrained optimization problem. Since $\alpha_{j}$ is between 0 and 1 , a suitable function is the sigmoid

$$
\alpha_{j}=\frac{1}{1+e^{-x_{j}}}, \quad j=1,2, \cdots, N_{e},
$$

where the range of $x_{j}$ is from $-\infty$ to $+\infty$. Now, Eq. (30) bocomes the unconstrained optimization problem

$$
\min f(\mathrm{x})=\frac{1}{2\left\|F_{\text {exp }}\right\|_{F}^{2}}\left[\sum_{i=1}^{m} \sum_{j=1}^{m}\left(F_{\text {ana }}(\mathbf{x})-F_{\text {exp }}\right)_{i j}^{2}\right]
$$

Subjected to : $-\infty<x_{j}<+\infty, \quad j=1,2, \cdots, N_{e}$,

where $\mathbf{x}=\left[x_{1}, x_{2}, \ldots, x_{N e}\right]^{T}$;

When using Gauss-Newton algorithm to solve the above problem, we need to compute the derivative of $f(\mathbf{x})$ with respect to $x_{k}$

$$
\begin{aligned}
\frac{\partial f(\mathbf{x})}{\partial x_{k}} & =\frac{1}{\left\|F_{\exp }\right\|_{F}^{2}}\left[\sum_{i=1}^{m} \sum_{j=1}^{m}\left(F_{\text {ana }}(\mathbf{x})-F_{\exp }\right)_{i j}\left(\frac{\partial F_{\text {ana }}(\mathbf{x})}{\partial x_{k}}\right)_{i j}\right] \\
& =\frac{1}{\left\|F_{\exp }\right\|_{F}^{2}}\left\{\operatorname{trace}\left[\left(F_{\text {ana }}(\mathbf{x})-F_{\text {exp }}\right)^{T}\left(\frac{\partial F_{\text {ana }}(\mathbf{x})}{\partial x_{k}}\right)\right]\right\} .
\end{aligned}
$$

Since $F_{\text {ana }}=\sum_{i=1}^{p} \phi_{i m} \lambda_{i}^{-1} \phi_{i m}^{T}$, where $\lambda_{i}=\omega_{i}^{2}, \omega_{i}$ is the $i$ th natural circular frequency, we have

$$
\begin{aligned}
\frac{\partial F_{\text {ana }}(\mathbf{x})}{\partial x_{k}} & =\sum_{i=1}^{p} \frac{\partial}{\partial x_{k}}\left(\phi_{i m} \lambda_{i}^{-1} \phi_{i m}^{T}\right) \\
& =\sum_{i=1}^{p}\left[\frac{\partial \phi_{i m}}{\partial x_{k}}\left(\lambda_{i}^{-1} \phi_{i m}^{T}\right)+\phi_{i m} \frac{\partial \lambda_{i}^{-1}}{\partial x_{k}}\left(\phi_{i m}^{T}\right)+\phi_{i m} \lambda_{i}^{-1} \frac{\partial \phi_{i m}^{T}}{\partial x_{k}}\right] \\
& =\sum_{i=1}^{p}\left[\frac{\partial \phi_{i m}}{\partial x_{k}}\left(\lambda_{i}^{-1} \phi_{i m}^{T}\right)-\phi_{i m} \lambda_{i}^{-1} \frac{\partial \lambda_{i}}{\partial x_{k}}\left(\lambda_{i}^{-1} \phi_{i m}^{T}\right)+\phi_{i m} \lambda_{i}^{-1} \frac{\partial \phi_{i m}^{T}}{\partial x_{k}}\right] .
\end{aligned}
$$

Now, the difficulty lies in how to compute the partial derivative of modes with respect to structural parameters, especially when the modes are incomplete. Since the dimension of complex 
civil structure is large, it will be computationally expensive to calculate all eigenvuales and eigenvectors of the system for each iteration. It is better to calculate the first few lower eigenpairs and use them to iteratively compute the derivative of eigenvectors with respect to structural variables. This problem has been studied by many researchers. Zhang \& Zerva's method (1996) is adopted in this paper. The eigenvalue derivative is

$$
\frac{\partial \lambda_{i}}{\partial x_{k}}=\phi_{i}^{T}\left[\frac{\partial K}{\partial x_{k}}-\lambda_{i} \frac{\partial M}{\partial x_{k}}\right] \phi_{i}
$$

Since we assume that mass matrix remains healthy and the definition $K=\sum_{k=1}^{N_{e}} \alpha_{k} K_{k}^{e}, \alpha_{k}=$ $\frac{1}{1+e^{-x_{k}}}, k=1,2, \ldots, N_{e}$, Eq. (35) can be simplified as

$$
\frac{\partial \lambda_{i}}{\partial x_{k}}=\phi_{i}^{T} \frac{\partial K}{\partial x_{k}} \phi_{i}=\phi_{i}^{T} \frac{\partial K}{\partial \alpha_{k}} \frac{\partial \alpha_{k}}{\partial x_{k}} \phi_{i}=\frac{e^{-x_{k}}}{\left(1+e^{-x_{k}}\right)^{2}} \phi_{i}^{T} K_{k}^{e} \phi_{i}
$$

Since $\phi_{i m}$ is the analytical modal shape of the structure at the measured DOFs, we can expand it to the complete modal shape vector. After we obtain the complete modal shape derivative, we can pick out the elements which correspond to the measured DOFs. Assume that the eigenvector derivative can be expressed as the linear combination of the first few modal shapes plus a static compensation term as

$$
\frac{\partial \phi_{i}}{\partial x_{k}}=\frac{\partial \phi_{i}}{\partial \alpha_{k}} \frac{\partial \alpha_{k}}{\partial x_{k}}=\left(\sum_{j=1}^{p} c_{j i} \phi_{j}+w_{i k}\right) \frac{e^{-x_{k}}}{\left(1+e^{-x_{k}}\right)^{2}},
$$

where the coefficient $c_{j i}$ can be obtained from the following equation

$$
\begin{gathered}
c_{j i}=\left\{\begin{array}{c}
\frac{-\phi_{j}^{T} F_{i}}{\lambda_{j}-\lambda_{i}}, \quad j \neq i \\
-0.5 \phi_{i}^{T} \frac{\partial M}{\partial \alpha_{k}} \phi_{i}, \quad j=i
\end{array}\right. \\
F_{i}=\left(\frac{\partial K}{\partial \alpha_{k}}-\frac{\partial \lambda_{i}}{\partial \alpha_{k}} M-\lambda_{i} \frac{\partial M}{\partial \alpha_{k}}\right) \phi_{i} .
\end{gathered}
$$

But the static compensation term $w_{i k}$ has to be computed iteratively by using the following procedure

$$
K\left(w_{i k}\right)_{l}=\left(I-F_{p}\right) F_{i}+\lambda_{i}\left(I-F_{p}\right) M\left(w_{i k}\right)_{l-1}, \quad l=1,2,3, \ldots,
$$

where $F_{p}$ is defines as

$$
F_{p}=\sum_{i=1}^{p} \phi_{i} \frac{1}{\omega_{i}^{2}} \phi_{i}^{T}
$$

Zhang \& Zerva (1996) proved that $\left(w_{i k}\right)_{l}$ will approach to the exact value when $l \rightarrow \infty$ and the convergence speed is inversely controlled by the ratio of $\lambda_{p} / \lambda_{p+1}$. This fact can help us to determine how many lower modes we need to compute in order to save computational time. 


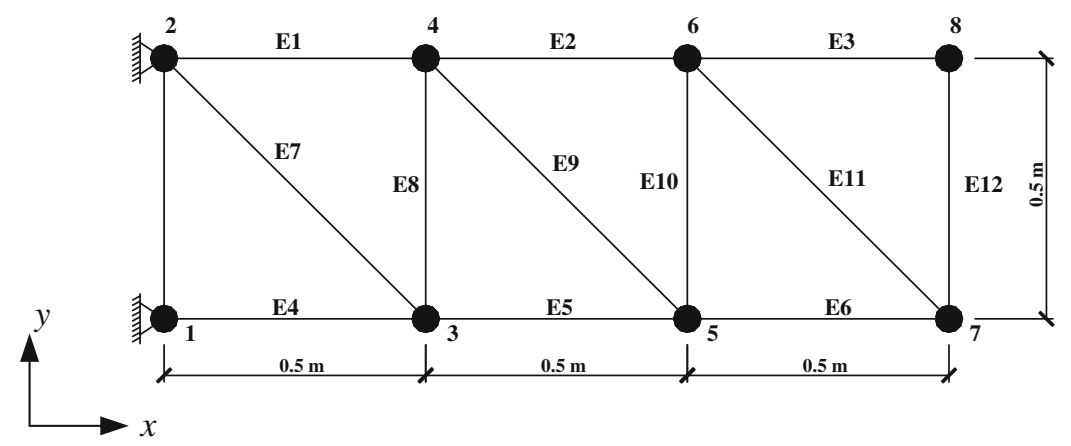

Figure 1. Three-bay aluminum truss structure.

After obtaining the derivatives of natural circular frequency and modal shapes, we can use Gauss-Newton method to solve the optimization problem in Eq. (32).

\section{Simulation examples}

\subsection{Three-bay cantilever truss structure}

As shown in figure 1, an analytical model of planar aluminum truss structure (Dharap et al 2006; Koh et al 2005) is considered to validate the damage detection method proposed in this paper. This three-bay planar truss model consists of 12 rod element connected at 8 nodes. The total length of the truss is $1.5 \mathrm{~m}$ with each bay being $0.5 \mathrm{~m}$ long and $0.5 \mathrm{~m}$ high, and rod element having Young's modulus of $E=70.3 \mathrm{GPa}$ and unit mass density of $\rho=2770 \mathrm{~kg} / \mathrm{m}^{3}$. All the members are 0.1 inch thick hollow tubes with outer diameter equal to 0.5 inch.

For experimental modal testing, measurement noise is unavoidable. Usually, the natural frequencies and mode shapes are reported (Farrar \& Cone 1995) most likely to be contaminated by 'measurement' noise, with a standard error of $\pm 0.15 \%$ and $\pm 3 \%$, respectively. In this example, the noisy mode shape is simulated as (Udwadia 2005)

$$
\widetilde{\phi}_{i}^{j}=\phi_{i}^{j}\left(1+\alpha_{\text {noise }} \xi\right)
$$

where $\phi_{i}^{j}$ is the $j$ th entry of the $i$ th modal shape; $\xi$ is a uniformly distributed random number between -1 and +1 ; and $\alpha_{\text {noise }}$ is the noise level, which is $3 \%$ for modal shapes and $1 \%$ for natural frequencies in this example.

Table 1. Damage scenarios for three-bay aluminum truss structure.

\begin{tabular}{llc}
\hline Damage cases & Damage location & $\begin{array}{c}\text { Stiffness reduction } \\
\text { factor }\left(1-\alpha_{j}\right)\end{array}$ \\
\hline 1. Single damage & Element No. 4 & 0.2 \\
2. Double damage & Element No. 1 & 0.2 \\
& Element No. 4 & 0.15 \\
3. Multiple damage & Element No. 1 & 0.2 \\
& Element No. 4 & 0.15 \\
& Element No. 9 & 0.25 \\
& Element No. 12 & 0.2 \\
\hline
\end{tabular}




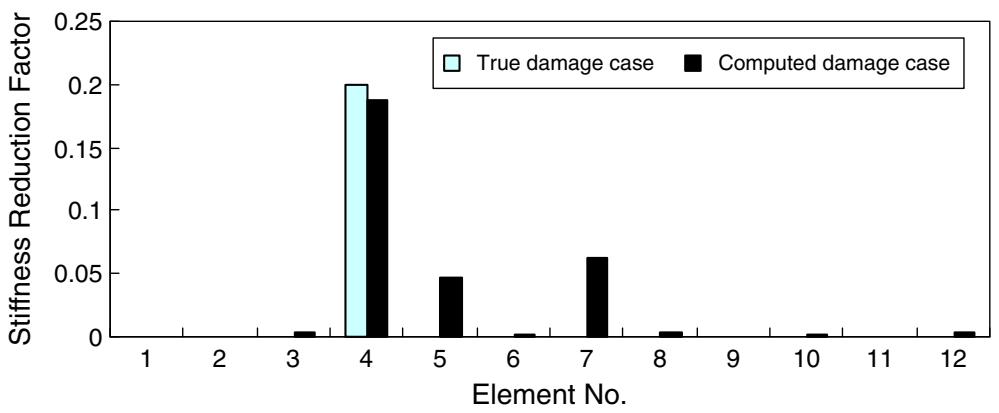

Figure 2. Damage location chart for single damage case.

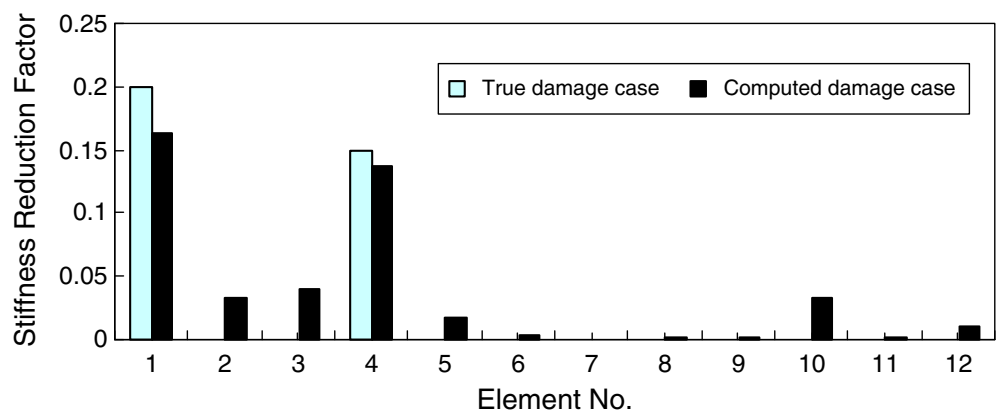

Figure 3. Damage location chart for double damage case.

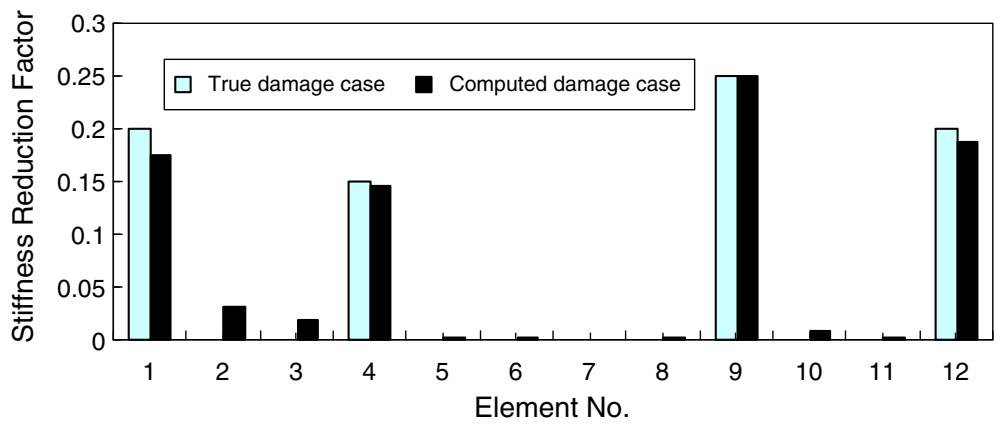

Figure 4. Damage location chart for multiple damage case.

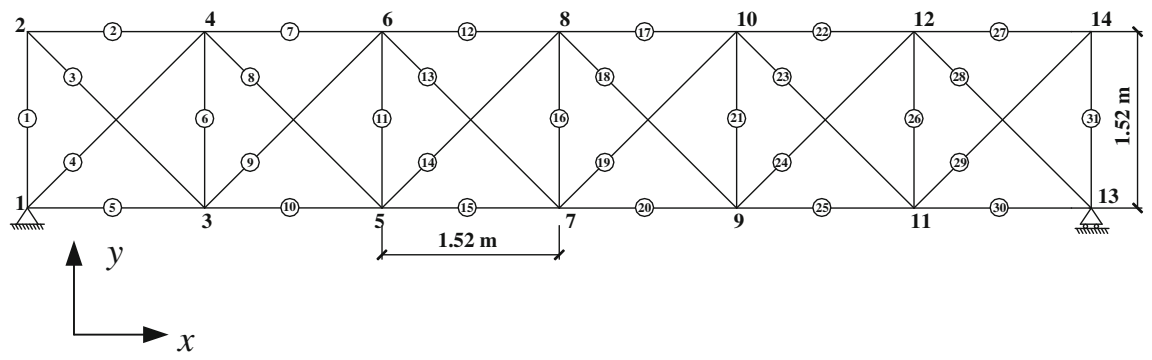

Figure 5. Six-bay truss structure. 
Table 2. Damage scenarios for six-bay truss structure.

\begin{tabular}{lcc}
\hline Damage cases & Damage location & $\begin{array}{c}\text { Stiffness reduction } \\
\text { factor }\left(1-\alpha_{j}\right)\end{array}$ \\
\hline 1. Single damage & Element No. 16 & 0.3 \\
2. Double damage & Element No. 1 & 0.3 \\
& Element No. 2 & 0.2 \\
\hline
\end{tabular}

Damage is simulated by reducing the stiffness of assumed elements. Three damage cases are considered, as shown in table 1 . Damage case 1 has $20 \%$ reduction in the stiffness in element 4; Damage case 2 has 20\% and 15\% reduction in elements 1 and 4; Damage case 3 has 20\%, 15\%, $25 \%$ and 20\% reduction in elements 1, 4, 9 and 12, respectively. Based on Kammer's method (Kammer 1991), the incomplete 'measured' mode shapes are selected at 10 sensor locations: 4 horizontal translation DOFs at nodes 5-8, and 6 vertical translations at nodes 3-8. Assume that only first 4 modal shapes are identified and used for structural damage detection. Damage location results for three damage cases are shown in figures 2,3 and 4 . Results show that the proposed method can detect the damaged elements existing in the structure using first few incomplete modal shapes and natural frequencies, though the computed damage magnitudes do not exactly match the true values due to measurement noise and computational error from modal parameter identification. Since the flexibility matrix is very sensitive to structural parameters, large noise in modal parameters will result in different damage pattern. It can be expected that increasing noise level in identified modal shapes and natural frequencies will lead to bigger mismatch between true damage patterns and computed damage patterns.

\subsection{Six-bay simple-supported truss structure}

The two-dimensional planar truss structure is shown in figure 5 (Shi et al 2000b). This truss consists of 31 elements and 14 nodes. The total length of the truss is $9.12 \mathrm{~m}$ with each bay being $1.52 \mathrm{~m}$ long and $1.52 \mathrm{~m}$ high, and rod element having Young's modulus of $E=70 \mathrm{GPa}$ and unit mass density of $\rho=2770 \mathrm{~kg} / \mathrm{m}^{3}$. Two damage cases are studied listing in table 2 . The identified modal parameters are simulated to contaminate $1 \%$ and $3 \%$ noise for natural frequencies and modal shapes, respectively. Based on Kammer's method (1991), the incomplete 'measured' mode shapes are selected at 16 sensor locations: 6 horizontal translation DOFs at

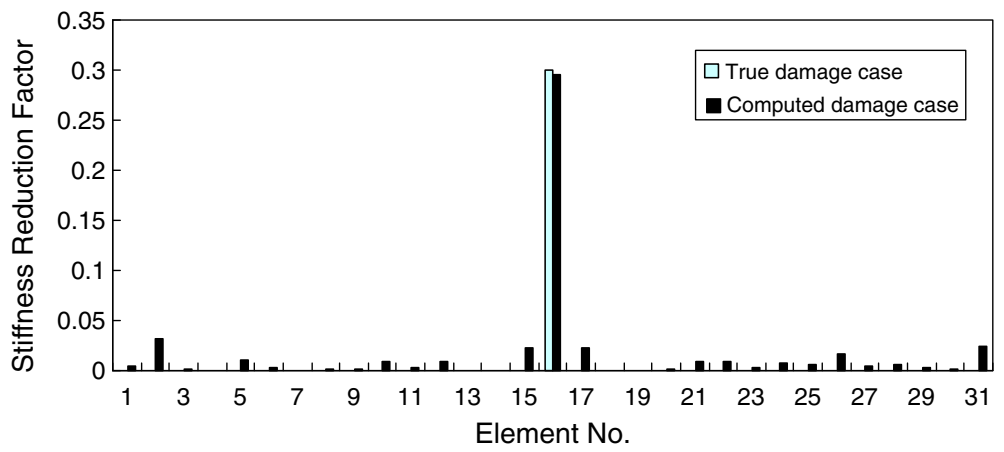

Figure 6. Damage location chart for damage case 1. 


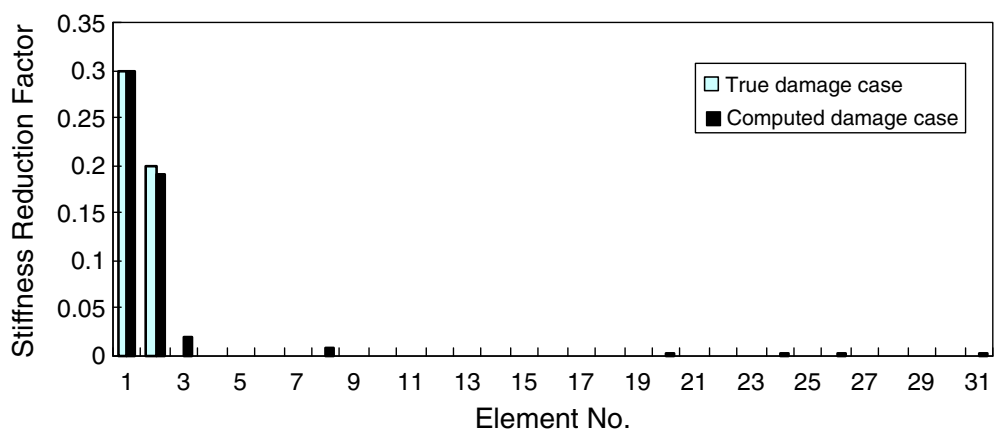

Figure 7. Damage location chart for damage case 2.

nodes $9-14$, and 10 vertical translations at nodes $3-12$. The damage identification results using the first 6 modal shapes are shown in figures 6 and 7. The simulation results show that the proposed method can successfully identify structural damages using the first few incomplete modal shapes and natural frequencies.

\section{Conclusions}

In this paper, the experimental modal flexibility matrix is obtained using incomplete first few modes. Then the optimization problem is constructed and solved by Gauss-Newton algorithm to minimize the difference between experimental modal flexibility matrix and analytical modal flexibility matrix. The optimal solution is closely related to structural physical parameters, which can be used for structural damage detection. Simulation examples show that the proposed method can successfully identify damaged elements in the structure, even if the identified modes are corrupted by light noise. However, since the proposed damage detection is a sensitivity-based method, the high noise level of the identified modes will affect the effectiveness of the proposed damage detection method. But, since the proposed method only requires the first few modal data that can be indentified more accurately with the advanced modern signal processing methods, the proposed method is still attractive. The proposed method will converge quickly because the rate of convergence of the Gauss-Newton algorithm with damping is linear.

\section{Acknowledgments}

The authors thank the support of the Texas Institute for the Intelligent Bio-Nano Materials and Structure for Aerospace Vehicles, funded by NASA Cooperative Agreement no. NCC-1-02038.

\section{References}

Bernal D 2002 Load vectors for damage localization. J. Eng. Mech., ASCE 128(1): 7-14

Bernal D 2006 Flexibility-based damage localization from stochastic realization results. J. Eng. Mech., ASCE 132(6): 651-658

Dharap P, Koh B H and Nagarajaiah S 2006 Structural health monitoring using ARMarkov observers. $J$. Intell. Mater. Syst. Struct. 17(6): 469-481

Doebling S W 1995 Measurement of structural flexibility matrices for experiments with incomplete reciprocity. Ph.D. Thesis, University of Colorado, Department of Aerospace Engineering Sciences 
Doebling S W, Farrar C R, Prime M B and Shevitz D W 1996 Damage Identification and health monitoring of structural and mechanical systems from changes in their vibration characteristics: A literature review. Research Rep. No. LA-13070-MS, ESA-EA, Los Alamos National Laboratory, NM

Farrar C R and Cone K M 1995 Vibration testing of the I-40 bridge before and after the introduction of damage. Proc. 13th Int. Modal Anal. Conf. 1: 203-209

Jaishi B and Ren W 2006 Damage detection by finite elment model updating using modal flexibility residual. J. Sound Vibr. 290(1-2): 369-387

Kammer D C 1991 Sensor placement for on-orbit modal identification and correlation of large space structures. J. Guidance 14(2): 251-259

Koh B H, Dharap P, Nagarajaiah S and Phan M Q 2005 Real-time structural damage monitoring by input error function. J. Amer. Inst. Aeronautics Astronautics, AIAA 43(8): 1808-1814

Liu P 1995 Identification and damage detection of trusses using modal data. J. Struct. Eng., ASCE 121(4): 599-608

Pandey A K and Biswas M 1994 Damage detection in structures using changes in flexibility. J. Sound Vibr. 169(1): 3-17

Ren W and Roeck G D 2002 Structural damage identification using modal data. I: simulation verification. J. Struct. Eng., ASCE 128(1): 87-95

Ricci S 2000 Best achievable modal eigenvectors in structural damage detection. Exp. Mech. 40(4): 425429

Shi Z Y, Law S S and Zhang L M 2000a Damage localization by directly using incomplete mode shapes. J. Eng. Mech., ASCE 126(6): 656-660

Shi Z Y, Law S S and Zhang L M 2000b Optimum sensor placement for structural damage detection. $J$. Eng. Mech., ASCE 126(11): 1173-1179

Sohn H, Farrar C R, Hemez F M, Shunk D D, Stinemates D W and Nadler B R 2003 A review of structural health monitoring literature: 1996-2001. Los Alamos National Laboratory Report, LA-13976-MS

Udwadia F E 2005 Structural identification and damage detection from noisy modal data. J. Aerospace Eng., ASCE 18(3): 179-187

Wu D and Law S S 2004 Model error correction from truncated model flexibility sensitivity and genetic parameters: Part I—simulation. Mech. Syst. Signal Process. 18: 1381-1399

Zhang O and Zerva A 1996 Iterative method for calculating derivatives of eigenvectors. AIAA J. 34(5): $1088-1090$

Zhao J and DeWolf J T 1999 Sensitivity study for vibration parameters used in damage detection. J. Struct. Eng., ASCE 125(4): 410-416 